\title{
Bridging the gap between current practice recommendations in national guidelines - a qualitative study of mental health services
}

\author{
Monica Stolt Pedersen ${ }^{1,2^{*}}$, Anne Landheim ${ }^{1,3}$, Lars Lien ${ }^{1,2}$ \\ From Health Services Research: Evidence-based practice \\ London, UK. 1-3 July 2014
}

\section{Background}

"National guideline for assessment, treatment and social rehabilitation of persons with concurrent substance use disorders and mental disorders", launched March 2012, is aimed at a wide range of health services. It holds a separate chapter on implementation.

The National Centre for Dual Diagnosis was commissioned by the Norwegian Directorate of Health to develop a plan for implementation of the Guideline. It contains tools to strengthen management, clinicians and consumers. A clinical audit tool was made to measure research-practice-gap. The clinical audit is the start-point of the implementation-plan, followed by an action schema. The implementation-process is as follows:

- Identify today's practice assessed against recommendations in the guideline, doing a clinical audit

- Identify areas of improvement based on the clinical audit
a) choose a goal for improvement
b) select initiatives based on goals
c) allocate responsibility
d) describe progress

\section{- Implementation phase}

- Evaluate by a new clinical audit and summarize experiences

This project aims to understand the process of using clinical audit as a basis for making choices aimed at clinical improvement in district psychiatric clinics. The objectives of the study are to describe and explore the implementation-process from the use of clinical audit to change in practice.

\section{Materials and methods}

Three different methods will be used, all qualitative, to explore the process: 1 ) observation of meetings, minutes of meetings etc. from the presentation of results from the clinical audit to a fully described action schema, 2) focusgroup interview with participants from each of the included units after second clinical audit, and 3) individual interviews with head of the units after second clinical audit. There is a desire to open up for experiences, perceptions, attitudes and narratives.

The setting is four units at a district psychiatric clinic, one outpatient clinic, two inpatient units, and one psychiatric outpatient emergency team.

\section{Results}

First observations are done and analysis pending. Preliminary results from the observation part of the study may be presented in July.

\section{Authors' details \\ ${ }^{1}$ National Centre for Dual Diagnosis, Innlandet Hospital Trust, Ottestad, Norway. ${ }^{2}$ Faculty of Medicine, University of Oslo, Oslo, Norway. ${ }^{3}$ Norwegian Centre for Addiction Research, University of Oslo, Oslo, Norway.}

Published: 7 July 2014

doi:10.1186/1472-6963-14-S2-P94

Cite this article as: Pedersen et al:: Bridging the gap between current practice recommendations in national guidelines - a qualitative study of mental health services. BMC Health Services Research 2014 14(Suppl 2):P94. 